\title{
THE ENDOMORPHISM SEMIRING OF A SEMILATTICE
}

\author{
J. JEŽEK, T. KEPKA AND M. MARÓTI
}

\begin{abstract}
We prove that the endomorphism semiring of a nontrivial semilattice is always subdirectly irreducible and describe its monolith. The endomorphism semiring is congruence simple if and only if the semilattice has both a least and a largest element.
\end{abstract}

\section{INTRODUCTION}

The explicit notion of a semiring (i.e., an algebra with two associative operations where one of them distributes over the other) was introduced by Vandiver [6] in 1934 (albeit, for instance, the semiring of natural numbers was commonly exploited long before that year). Congruence-simple structures (i.e., those possessing just two congruence relations) were and are studied in many classes of algebras and in some cases they are quite popular (e.g., simple groups or rings). On the other hand, not much is known about congruence-simple semirings ([1],[2],[4],[5] and[7]).

If $S$ is a congruence-simple semiring with commutative addition, then we get a natural homomorphism $\varphi$ of $S$ into the endomorphism semiring of the additive (commutative) semigroup of $S$. Since $S$ is simple, either $\operatorname{ker}(\varphi)=S \times S$ (a rather trivial case) or $\operatorname{ker}(\varphi)=\mathbf{i d}_{S}$ and then $S$ is embedded into the endomorphism semiring. Thus in order to get representations of simple semirings with commutative addition, we have to consider the question of simplicity of endomorphism semirings of commutative semigroups. In the present paper we are concerned with the particular case when the commutative semigroup is idempotent, i.e., a semilattice. A similar question is considered in [7] for semilattices with neutral elements.

By a semilattice we will mean a join semilattice, i.e., an algebra with one binary operation $\vee$ satisfying the identities $x \vee(y \vee z)=(x \vee y) \vee z$, $x \vee y=y \vee x$ and $x \vee x=x$. Thus, for elements $x, y$ of a semilattice $M$, $x \leq y$ means that $x \vee y=y$. In this notation, a neutral element is the least element; of course, it does not need to exist. The set $E$ of endomorphisms of $M$ is a semiring with respect to the addition and multiplication defined as follows: $f+g=h$ where $h(x)=f(x) \vee g(x)$ for all $x \in M ; f g=h$ where

1991 Mathematics Subject Classification. 06A12, 16Y60.

Key words and phrases. semilattice, semiring, subdirectly irreducible, simple.

The work is a part of the research project MSM0021620839 financed by MSMT. The third author was partially supported by the Hungarian National Foundation for Scientific Research (OTKA), grant nos. T 48809 and K 60148. 
$h(x)=f(g(x))$ for all $x \in M$. This semiring $E$ will be denoted by $\mathbf{E}_{M}$ and called the endomorphism semiring of $M$.

For every element $a \in M$ denote by $\bar{a}$ the element of $\mathbf{E}_{M}$ with $\bar{a}(x)=a$ for all $x \in M$. These are the constant endomorphisms of $M$. For every triple $a, b, c$ of elements of $M$ with $a \leq b$ denote by $\rho_{a, b, c}$ the mapping of $M$ into itself defined by $\rho_{a, b, c}(x)=a$ if $x \leq c$ and $\rho_{a, b, c}(x)=b$ if $x \not \leq c$. Clearly, $\rho_{a, b, c} \in \mathbf{E}_{M}$. We have $\bar{a}=\rho_{a, a, c}$ for any $c \in M$. The subsemiring of $\mathbf{E}_{M}$ generated by its elements $\rho_{a, b, c}$ (where $a \leq b$ and $c$ is arbitrary) will be denoted by $\mathbf{F}_{M}$. Notice that $\mathbf{F}_{M}$ is a left ideal of the semiring $\mathbf{E}_{M}$ and it is an ideal, provided that $M$ is finite.

The aim of this paper is to prove that the endomorphism semiring $\mathbf{E}_{M}$ of any nontrivial semilattice $M$ is subdirectly irreducible, to describe the monolith of the semiring and to characterize the semilattices $M$ such that the endomorphism semiring of $M$ is (congruence) simple. Also, we will do the same for any subsemiring of $\mathbf{E}_{M}$ containing $\mathbf{F}_{M}$.

Let us recall that an algebra $A$ is said to be subdirectly irreducible if it is nontrivial (i.e., has at least two elements) and among its non-identity congruences there exists the least one; this least non-identity congruence of $A$ is then called the monolith of $A$. An algebra is called simple if it is nontrivial and has only two congruences (the identity $\mathbf{i d}_{A}$ and the all-relation $A \times A)$.

The reader is referred to [3] for these and other facts on semilattices and universal algebra.

\section{The MONOLITH}

2.1. Theorem. Let $M$ be a nontrivial semilattice and $E$ be a subsemiring of the endomorphism semiring $\mathbf{E}_{M}$ such that $\mathbf{F}_{M} \subseteq E$. Then $E$ is subdirectly irreducible and its monolith $\mu$ can be described as follows: for $f, g \in E$, $(f, g) \in \mu$ if and only if either $f=g$ or the following three conditions are satisfied:

(1) the range of $f$ is a lower bounded subset of $M$;

(2) the range of $g$ is a lower bounded subset of $M$;

(3) there exists an $a \in M$ such that $f(x) \vee a=g(x) \vee a$ for all $x \in M$.

Proof. Denote by $R$ the set of the ordered pairs $(f, g) \in E \times E$ satisfying either $f=g$ or the three conditions. We are going to prove that $R$ is a congruence of $E$.

Clearly, $R$ is a reflexive and symmetric relation on $E$.

Let $(f, g) \in R$ and $(g, h) \in R$; we need to show that $(f, h) \in R$. This is clear if either $f=g$ or $g=h$. Otherwise, the ranges of $f, g, h$ are all lower bounded and there exist elements $a, b \in M$ such that $f(x) \vee a=g(x) \vee a$ and $g(x) \vee b=h(x) \vee b$ for all $x \in M$. We have $f(x) \vee(a \vee b)=g(x) \vee a \vee b=$ $h(x) \vee(a \vee b)$ for all $x \in M$ and thus $(f, h) \in R$.

Thus $R$ is an equivalence on $E$. Let $(f, g) \in R$ and $h \in E$; we need to show that $(f+h, g+h) \in R,(f h, g h) \in R$ and $(h f, h g) \in R$. It is sufficient 
to consider the case when $f \neq g$. There exist elements $a, b, c \in M$ such that $f(x) \geq a, g(x) \geq b$ and $f(x) \vee c=g(x) \vee c$ for all $x \in M$.

We have $(f+h)(x) \geq a,(g+h)(x) \geq b$ and $(f+h)(x) \vee c=(g+h)(x) \vee c$ for all $x \in M$, so that $(f+h, g+h) \in R$.

We have $(f h)(x) \geq a,(g h)(x) \geq b$ and $(f h)(x) \vee c=f(h(x)) \vee c=$ $g(h(x)) \vee c=(g h)(x) \vee c$ for all $x \in M$, so that $(f h, g h) \in R$.

We have $(h f)(x) \geq h(a),(h g)(x) \geq h(b)$ and $(h f)(x) \vee h(c)=h(f(x) \vee c)=$ $h(g(x) \vee c)=(h g)(x) \vee h(c)$ for all $x \in M$, so that $(h f, h g) \in R$.

Thus $R$ is a congruence of $E$.

It is clear that $(\bar{a}, \bar{b}) \in R$ for all $a, b \in M$. Thus $R \neq \mathbf{i d}_{M}$, since $M$ is nontrivial. We are going to prove that $R$ is the monolith of $E$.

Let $S$ be any congruence of $E$ such that $S \neq \mathbf{i d}_{M}$. There exists a pair $(f, g) \in S$ with $f \neq g$. We have $f(a) \neq g(a)$ for an element $a \in M$; we can suppose without loss of generality that $g(a) \not \leq f(a)$. (Otherwise, we could consider the pair $(g, f)$ instead of the pair $(f, g)$.)

Let $b_{1}, b_{2}$ be any elements of $M$ such that $b_{1} \leq b_{2}$. The endomorphism $h=\rho_{b_{1}, b_{2}, f(a)}$ belongs to $E$, as well as all constant endomorphisms. Since $S$ is a congruence, we have $(h f \bar{a}, h g \bar{a}) \in S$. But $h f \bar{a}=\overline{b_{1}}$ and $h g \bar{a}=\overline{b_{2}}$. Thus any two comparable constants are $S$-related.

Let $c_{1}, c_{2}$ be any two elements of $M$; put $c=c_{1} \vee c_{2}$. Then $\left(\overline{c_{1}}, \bar{c}\right) \in S$ and $\left(\bar{c}, \overline{c_{2}}\right) \in S$, so that $\left(\overline{c_{1}}, \overline{c_{2}}\right) \in S$. Thus any two constants are $S$-related.

Let $(p, q) \in R$ and $p \neq q$. There exist elements $a_{1}, a_{2}, e \in M$ such that $p(x) \geq a_{1}, q(x) \geq a_{2}$ and $p(x) \vee e=q(x) \vee e$ for all $x \in M$. Since $\left(\overline{a_{1}}, \bar{e}\right) \in S$ and $S$ is a congruence, we have $\left(\overline{a_{1}}+p, \bar{e}+p\right) \in S$, i.e., $(p, \bar{e}+p) \in S$. Similarly, since $\left(\overline{a_{2}}, \bar{e}\right) \in S$, we have $\left(\overline{a_{2}}+q, \bar{e}+q\right) \in S$, i.e., $(q, \bar{e}+q) \in S$. But $\bar{e}+p=\bar{e}+q$ and so $(p, q) \in S$.

We have shown that $R \subseteq S$ for any congruence $S$ of $E$ other than $\mathbf{i d}_{M}$. Thus $R$ is the monolith of $E$ and $E$ is subdirectly irreducible.

2.2. Remark. Let $M$ be a nontrivial semilattice with the least element 0 . Put $\mathbf{E}_{M, 0}=\left\{f \in \mathbf{E}_{M}: f(0)=0\right\}$ and denote by $\mathbf{F}_{M, 0}$ the subsemiring of $\mathbf{E}_{M, 0}$ generated by all $\rho_{0, b, c}$ with $b, c \in M$. Proceeding similarly as in the proof of 2.1 we can show that every subsemiring $E_{0}$ such that $\mathbf{F}_{M, 0} \subseteq E_{0} \subseteq \mathbf{E}_{M, 0}$ is subdirectly irreducible and a pair $(f, g)$ belongs to its monolith if and only if either $f=g$ or the condition 2.1(3) is satisfied.

\section{Simplicity}

3.1. Theorem. Let $M$ be a nontrivial semilattice and $E$ be a subsemiring of the endomorphism semiring $\mathbf{E}_{M}$ such that $\mathbf{F}_{M} \subseteq E$.

(1) If $M$ has both a least and a largest element, then $E$ is simple.

(2) If $E$ is simple and $\mathbf{i d}_{M} \in E$, then $M$ has both a least and a largest element.

Proof. By 2.1, $E$ is simple if and only if the monolith $R$ of $E$ described there is equal to $E \times E$. If $M$ has both a least and a largest element, then it is 
clear that $R=E \times E$. If $M$ has no least element then $\left(\mathbf{i d}_{M}, \bar{a}\right) \notin R$ for any constant endomorphism $\bar{a}$ of $E$. If $M$ has the least element $0_{M}$ but no largest element, then $\left(\mathbf{i d}_{M}, \bar{a}\right) \notin R$ for any $a \in M-\left\{0_{M}\right\}$.

3.2. Corollary. Let $M$ be a nontrivial semilattice. Then $\mathbf{E}_{M}$ is simple if and only if $M$ has both a least and a largest element.

3.3. Corollary. Let $M$ be a nontrivial finite semilattice. Then $\mathbf{E}_{M}$ is simple if and only if $M$ is a lattice.

3.4. Theorem. The semiring $\mathbf{F}_{M}$ is simple for any nontrivial semilattice $M$.

Proof. Put $E=\mathbf{F}_{M}$. By 2.1, it is sufficient to prove that the monolith $R$ of $E$ equals $E \times E$. By a rho we will mean an endomorphism $\rho_{a, b, c}$ of $M$ for some $a, b, c \in M$ with $a \leq b$. One can see easily that the product of two rhos is a rho; since $E$ is generated by the rhos, it follows that every element of $E$ is a sum of finitely many rhos. From the description of $R$ it follows that any two rhos are $R$-equivalent. Then also any two finite sums of rhos are $R$-equivalent.

3.5. Remark. Let $M$ be a nontrivial semilattice and let $E$ be a subsemiring of $\mathbf{E}_{M}$ such that $\mathbf{F}_{M} \subseteq E$.

(1) If $M$ has the least element (say 0), then $\overline{0} \in E$ and it follows from 2.1 that $E$ is simple if and only if the range of $f$ is an upper bounded subset of $M$ for every $f \in E$.

(2) If $M$ has the largest element (say 1), then $\overline{1} \in E$ and it follows from 2.1 that $E$ is simple if and only if the range of $f$ is a lower bounded subset of $M$ for every $f \in E$.

3.6. Remark. Let $M$ be a nontrivial semilattice with the least element 0 . Using 2.2 and going through the proofs of $3.1,3.2,3.3$ and 3.4 , we check easily that similar results remain true for subsemirings $E$ such that $\mathbf{F}_{M, 0} \subseteq$ $E \subseteq \mathbf{E}_{M, 0}$. Some of these results are proved in [7].

\section{When $\mathbf{F}_{M}=\mathbf{E}_{M}$}

Let $M$ be a semilattice. An element $a$ of $M$ is said to be pseudoirreducible (in $M$ ) if $x \vee y=a$ implies $a \in\{x, y\}$. An element $a$ of $M$ is said to be splitting (in $M$ ) if the set $\{x \in M: x \nsupseteq a\}$ has the largest element. (That is, if there exists an element $b$ such that $b \nsupseteq a$ and for every $x \in M$, either $x \geq a$ or $x \leq b$.) Clearly, every splitting element of $M$ is pseudoirreducible.

4.1. Lemma. A finite lattice $M$ is distributive if and only if every pseudoirreducible element of $M$ is either splitting or the least element of $M$.

Proof. The direct implication is evident. The proof of the converse can be based on the well-known duality between finite distributive lattices and finite ordered sets: as it is easy to check, $M$ is (under the assumption) isomorphic to the distributive lattice of order ideals of the ordered subset of $M$ consisting of the pseudoirreducible elements of $M$ with the least element excluded. 
4.2. Theorem. The following four conditions are equivalent for a semilattice $M$ :

(1) $\mathbf{F}_{M}=\mathbf{E}_{M}$;

(2) the semiring $\mathbf{F}_{M}$ has a left (right, respectively) multiplicatively neutral element;

(3) the semiring $\mathbf{F}_{M}$ has a multiplicatively neutral element;

(4) $M$ is a finite distributive lattice.

Proof. Let $M$ be nontrivial. If $f \in \mathbf{F}_{M}$ is a left multiplicatively neutral element (i.e., $f g=g$ for all $g \in \mathbf{F}_{M}$ ), then $f(a)=f \bar{a}(a)=\bar{a}(a)=a$ for every $a \in M$, so that $f=\mathbf{i d} \mathbf{d}_{M}$. If $g \in \mathbf{F}_{M}$ is a right multiplicatively neutral element and $a, b, x \in M$ where $a<b$, then $a=\rho_{a, b, g(x)}(g(x))=\rho_{a, b, g(x)}(x)$, so that $x \leq g(x)$; similarly, $a=\rho_{a, b, x}(x)=\rho_{a, b, x}(g(x))$, so that $g(x) \leq x$; we get $g=\mathbf{i d}_{M}$ also in this case. It follows that it suffices to prove the equivalence of the conditions (1) and (4).

Let $\mathbf{F}_{M}=\mathbf{E}_{M}$. Then every element of $\mathbf{E}_{M}$ can be expressed as the sum of finitely many elements $\rho_{a, b, c}$ with $a, b, c \in M$ and $a \leq b$. In particular, $\operatorname{id}_{M}=\rho_{a_{1}, b_{1}, c_{1}}+\cdots+\rho_{a_{n}, b_{n}, c_{n}}$ for some $n \geq 1$ and some elements $a_{i}, b_{i}, c_{i}$ with $a_{i} \leq b_{i}$. Since the range of that join is finite, the range of $\mathbf{i d}_{M}$ must be finite and thus $M$ is finite. Since the range of the join is lower bounded, the range of $\mathbf{i d}_{M}$ is lower bounded and thus $M$ has the least element. A finite join semilattice with a least element is a lattice. Denote the least element by $0_{M}$. Since $\mathbf{i d}_{M}\left(0_{M}\right)=0_{M}$, we have $a_{1}=\cdots=a_{n}=0_{M}$. Then we can suppose that $b_{i} \neq 0_{M}$ for all $i$.

Let $a$ be a pseudoirreducible element of $M, a \neq 0_{M}$. From

$$
a=\mathbf{i d}_{M}(a)=\rho_{0_{M}, b_{1}, c_{1}}(a) \vee \cdots \vee \rho_{0_{M}, b_{n}, c_{n}}(a)
$$

it follows that $a=\rho_{0_{M}, b_{i}, c_{i}}(a)$ for some $i$. But then $a \not \leq c_{i}$ and $a=b_{i}$. Since $\rho_{0_{M}, b_{i}, c_{i}}(x) \leq x$ for all $x \in M$, we get that $x \not \leq c_{i}$ implies $b_{i} \leq x$. These facts together mean that $a$ is a splitting element of $M$. Thus every pseudoirreducible element of $M$ other than $0_{M}$ is splitting, so that $M$ is distributive according to 4.1 .

We have proved that (1) implies (4). In order to prove the converse, let $M$ be a nontrivial finite distributive lattice. Denote by $b_{1}, \ldots, b_{n}$ all pseudoirreducible elements of $M$ other than $0_{M}$. By 4.1, for every $i$ there exists an element $c_{i} \in M$ such that $c_{i} \nsupseteq b_{i}$ and for every $x \in M$, either $x \geq b_{i}$ or $x \leq c_{i}$. Put $f=\rho_{0_{M}, b_{1}, c_{1}}+\cdots+\rho_{0_{M}, b_{n}, c_{n}}$, so that $f \in \mathbf{F}_{M}$.

For every $i$ we have $f\left(b_{i}\right)=b_{i}$. To see this, observe first that $\rho_{0_{M}, b_{i}, c_{i}}\left(b_{i}\right)=$ $b_{i}$ (since $\left.b_{i} \not \leq c_{i}\right)$. Now let $j \neq i$. If $b_{i} \leq c_{j}$ then $\rho_{0_{M}, b_{j}, c_{j}}\left(b_{i}\right)=0_{M} \leq b_{i}$. In the remaining case, when $b_{i} \not \leq c_{j}$, we have $b_{i} \geq b_{j}$ and thus $\rho_{0_{M}, b_{j}, c_{j}}\left(b_{i}\right)=$ $b_{j} \leq b_{i}$. Thus $f\left(b_{i}\right)$ is the join of several elements, each of which is less or equal $b_{i}$ and at least one of them is equal to $b_{i}$.

Since every element of $M$ is the join of some pseudoirreducible elements, it follows that $f(x)=x$ for all $x \in M$, i.e., $f=\mathbf{i d}_{M}$. Thus $\operatorname{id}_{M} \in \mathbf{F}_{M}$. Since $\mathbf{F}_{M}$ is a left ideal of the semiring $\mathbf{E}_{M}$, it follows that $\mathbf{F}_{M}=\mathbf{E}_{M}$. 
4.3. Remark. Let $M$ be a semilattice with the least element 0 . Theorem 4.2 remains true for the subsemirings $\mathbf{F}_{M, 0}$ and $\mathbf{E}_{M, 0}$ (see 2.2). In the finite case, the main part of this result is proved in $[7,4.9,4.10]$.

\section{REFERENCES}

[1] R. El Bashir, J. Hurt, A. Jančařík and T. Kepka, Simple commutative semirings. J. Algebra 235 (2001), 277-306.

[2] R. El Bashir and T. Kepka, Congruence-simple semirings (preprint).

[3] R. McKenzie, G. McNulty and W. Taylor, Algebras, Lattices, Varieties, Volume I. Wadsworth \& Brooks/Cole, Monterey, CA, 1987.

[4] S. S. Mitchell and P. B. Fenoglio, Congruence-free commutative semirings. Semigroup Forum 37 (1988), 79-91.

[5] C. Monico, On finite congruence-simple semirings. J. Algebra 271 (2004), 846-854.

[6] H. S. Vandiver, Note on a simple type of algebras in which the cancellation law of addition does not hold. Bull. Amer. Math. Soc. 40 (1934), 916-920.

[7] J. Zumbrägel, Classification of finite congruence-simple semirings with zero (preprint).

MFF UK, Sokolovská 83, 18600 Praha 8, Czech Republic

E-mail address: jezek@karlin.mff.cuni.cz

E-mail address: kepka@karlin.mff.cuni.cz

Bolyai Institute, University of Szeged, Szeged, Hungary

E-mail address: mmaroti@math.u-szeged.hu 